\title{
p38MAPK is a novel DNA damage response-independent regulator of the senescence-associated secretory phenotype
}

\section{Adam Freund ${ }^{1,2,3}$, Christopher K Patil ${ }^{2,3}$ and Judith Campisi ${ }^{2,3, *}$}

${ }^{1}$ Department of Molecular and Cell Biology, University of California, Berkeley, CA, USA, ${ }^{2}$ Buck Institute for Age Research, Novato, CA, USA and ${ }^{3}$ Life Sciences Division, Lawrence Berkeley National Laboratory, Berkeley, CA, USA

Cellular senescence suppresses cancer by forcing potentially oncogenic cells into a permanent cell cycle arrest. Senescent cells also secrete growth factors, proteases, and inflammatory cytokines, termed the senescence-associated secretory phenotype (SASP). Much is known about pathways that regulate the senescence growth arrest, but far less is known about pathways that regulate the SASP. We previously showed that DNA damage response (DDR) signalling is essential, but not sufficient, for the SASP, which is restrained by $\mathrm{p} 53$. Here, we delineate another crucial SASP regulatory pathway and its relationship to the DDR and p53. We show that diverse senescenceinducing stimuli activate the stress-inducible kinase p38MAPK in normal human fibroblasts. p38MAPK inhibition markedly reduced the secretion of most SASP factors, constitutive p38MAPK activation was sufficient to induce an SASP, and p53 restrained p38MAPK activation. Further, p38MAPK regulated the SASP independently of the canonical DDR. Mechanistically, p38MAPK induced the SASP largely by increasing NF-кB transcriptional activity. These findings assign p38MAPK a novel role in SASP regulation-one that is necessary, sufficient, and independent of previously described pathways.

The EMBO Journal (2011) 30, 1536-1548. doi:10.1038/

emboj.2011.69; Published online 11 March 2011

Subject Categories: signal transduction; genome stability \& dynamics

Keywords: aging; cancer; inflammation; NF-кB; tumour suppression

\section{Introduction}

Cellular senescence halts the proliferation of cells at risk for malignant transformation. Many potentially oncogenic stimuli, ranging from DNA damage to the activation of certain oncogenes, can induce a senescence response (Campisi and d'Adda di Fagagna, 2007). Recent data showing that cellular

\footnotetext{
*Corresponding author. The Buck Institute for Age Research, 8001 Redwood Blvd, Novato, CA 94945, USA.

Tel.: + 1415209 2066; Fax: + 1415493 3640;

E-mail: jcampisi@buckinstitute.org
}

Received: 21 October 2010; accepted: 18 February 2011; published online: 11 March 2011 senescence is a common response to oncogene activation in vivo (Prieur and Peeper, 2008) suggest that senescence may be as important as apoptosis for suppressing the development of cancer.

The senescent phenotype is multi-faceted. The chief hallmark of senescent cells-the halt of proliferation (hereafter, growth arrest) - is essentially irreversible in that it cannot be reversed by physiological stimuli. This growth arrest is established and maintained by the p53 and $\mathrm{p} 16^{\mathrm{INK} 4 \mathrm{a}} / \mathrm{pRB}$ pathways. Although many questions remain, we broadly understand the interwoven and complementary mechanisms by which these pathways regulate the growth arrest (Collins and Sedivy, 2003; Ohtani et al, 2004; Gil and Peters, 2006; Campisi and d'Adda di Fagagna, 2007; Rodier et al, 2007). By contrast, we understand little about how other senescenceassociated phenotypes are controlled.

Senescent cells develop an enlarged morphology, upregulate a senescence-associated $\beta$-galactosidase (SA- $\beta$ gal) activity (Dimri et al, 1995), and show widespread changes in chromatin organization and gene expression (Campisi and d'Adda di Fagagna, 2007). Of particular biological importance, senescent cells increase the expression and secretion of numerous cytokines, chemokines, matrix metalloproteinases (MMPs), and other proteins that can alter local tissue environments. We termed this feature the senescenceassociated secretory phenotype (SASP) (Coppe et al, 2008, 2010b).

The SASP can be beneficial or deleterious, depending on the biological context. Among the benefits, some SASP components reinforce the senescence growth arrest (Kortlever et al, 2006; Acosta et al, 2008; Kuilman et al, 2008; Wajapeyee et al, 2008). Others signal the immune system to clear senescent cells (Xue et al, 2007), and SASP MMPs can suppress fibrotic scar formation (Krizhanovsky et al, 2008; Jun and Lau, 2010). Among the deleterious effects, SASP MMPs can disrupt mammary morphogenesis in cell culture models (Parrinello et al, 2005). Further, in culture and in vivo, SASP factors can promote malignant phenotypes, including cell proliferation (Bavik et al, 2006; Coppe et al, 2010b), angiogenesis (Coppe et al, 2006), epithelial-to-mesenchymal transitions and invasiveness (Coppe et al, 2008), and accelerated growth of xenografted tumours (Krtolica et al, 2001; Liu and Hornsby, 2007). Moreover, many SASP factors are pro-inflammatory. Thus, senescent cells, which increase with age in vivo (Dimri et al, 1995; Paradis et al, 2001; Jeyapalan et al, 2007; Zhou et al, 2008), may contribute to the low-level chronic inflammation that is a hallmark of aged mammalian tissues and many age-related diseases (Freund et al, 2010; Coppe et al, 2010a). This important anti-cancer defense, then, might be antagonistically pleiotropic and, ironically, promote cancer in aged tissues. Given the known and proposed importance of the SASP, it is crucial to understand its regulation. 
Although the senescence growth arrest and SASP are often coordinately induced, the pathways that regulate them do not completely overlap. For example, p16 $6^{\mathrm{INK} 4 \mathrm{a}}$ expression is sufficient to induce a senescence growth arrest, but does not induce or modify the SASP (Coppe et al, 2010a). Likewise, p53 is required for the growth arrest (Campisi and d'Adda di Fagagna, 2007; Courtois-Cox et al, 2008), but not the SASP; in fact, cells lacking functional p53 secrete markedly higher levels of most SASP factors. Thus, p53 restrains the SASP (Coppe et al, 2008), suggesting it suppresses tumorigenesis in part by limiting the development of a pro-inflammatory tissue environment. The pathways by which p53 restrains the SASP are not known.

The SASP is not an acute (rapid, transient) inflammatory response. It does not develop immediately after cells experience a senescence-inducing stimulus but, once established, it persists for long intervals (Coppe et al, 2008, 2010b; Rodier et al, 2009). One regulator of the SASP is the DNA damage response (DDR), the signalling cascade that senses and ultimately repairs DNA damage. The DDR is initiated by phosphatidylinositol 3-kinase-like protein kinases such as ATM and ATR, which phosphorylate chromatin modifying proteins such as $\gamma \mathrm{H} 2 \mathrm{AX}$, adaptor proteins such as NBS1, and downstream kinases such as CHK1 and CHK2, which ultimately activate p53 (Ciccia and Elledge, 2010). ATM, CHK2, and NBS1 are essential for establishing and maintaining the expression of several SASP proteins, particularly inflammatory cytokines such as IL-6 and IL-8 (Rodier et al, 2009). However, canonical DDR signalling is not sufficient for the SASP: a transient DDR, caused by low-level ionizing radiation, does not induce an SASP (Rodier et al, 2009). Additionally, the SASP, like some other features of the senescent phenotype (e.g., cell enlargement, SA- $\beta$ gal activity), takes several days to develop after the damaging event, whereas the canonical DDR is activated immediately after damage. Thus, at least one additional, slower event-which cooperates with the DDR, but is independent of rapidresponse DDR factors-must be required for the SASP.

Here, we show that this event is activation of p38MAPK, a member of the mitogen-activated protein kinase (MAPK) family. Like other MAPK members, p38MAPK is activated by phosphorylation, and this generally occurs rapidly (within minutes) and transiently (subsiding within a few hours) in response to acute cellular stress (Cuenda and Rousseau, 2007). p38MAPK is important for the senescence growth arrest due to its ability to activate both the p53 and pRb/ p16 growth arrest pathways. p38MAPK inhibition moderately delays replicative senescence (caused by dysfunctional telomeres, which resemble DNA double-strand breaks) (Iwasa et al, 2003), and the rapid senescence of cells from patients with Werner's syndrome, a premature aging disorder caused by a defective DNA repair protein (Davis and Kipling, 2009). Further, p38MAPK activity is required for the senescence arrest caused by oncogenic RAS, and constitutive p38MAPK activity can induce a growth arrest in normal human cells (Wang et al, 2002; Deng et al, 2004). Whether p38MAPK regulates the SASP had not been explored. p38MAPK is known to upregulate specific cytokines such as IL-6, IL-8, and TNF $\alpha$ in some biological contexts (Ono and Han, 2000; Zhang et al, 2007), but the upregulation is generally an acute response, whereas the SASP is chronic and includes $>40$ secreted proteins comprising cytokines, chemokines, growth factors, MMPs and shed receptors and ligands (Coppe et al, 2008, 2010b).

We show that p38MAPK activity is necessary and sufficient for development of an SASP in cells induced to senesce by direct DNA damage or oncogenic RAS. In these contexts, p38MAPK is not activated rapidly, but rather with delayed kinetics characteristic of the SASP. Further, p53 restrains the SASP by restraining p38MAPK activation, and p38MAPK activation occurs independently of the DDR. Further, p38MAPK regulates the SASP mainly through NF- $\kappa B$ transcriptional activity, which we show is required for the expression of most SASP factors. These findings assign the p38MAPK pathway a novel role in senescence regulation.

\section{Results}

\section{p38MAPK is activated during the senescence response to genotoxic stress}

p38MAPK is activated by tyrosine and threonine phosphorylation in response to a variety of stresses (Cuenda and Rousseau, 2007), including oncogenic RAS (Ha-RAS ${ }^{\mathrm{V} 12}$ ) expression (Wang et al, 2002), which indirectly causes DNA damage (Di Micco et al, 2006). To determine whether p38MAPK activation is a direct genotoxic stress response, we X-irradiated (XRA; $10 \mathrm{~Gy}$ ) presenescent (PRE) normal human fibroblasts (strain HCA2) to synchronously induce senescence (SEN(XRA)). After XRA, cells were cultured for 10 days, during which time they developed classic markers of senescence: growth arrest (no increase in cell number; low 5-bromodeoxyuridine (BrdU) labeling), an enlarged flattened morphology, and SA- $\beta$ gal activity (Supplementary Figures S1A and B; not shown). To assess p38MAPK activation, we prepared whole cell lysates at intervals after XRA until cells developed a complete senescent phenotype 8-10 days later. We analysed levels of total and phosphorylated p38MAPK and its downstream target Hsp27 (Beyaert et al, 1996; Davis et $a l, 2005)$ by western blot. Unlike the rapid and robust response to LPS, p38MAPK phosphorylation (p38-P) increased only slightly in the $24 \mathrm{~h}$ immediately after XRA (Supplementary Figure S1C). p38-P and Hsp27-P levels did not rise substantially until 2-4 days after XRA, reaching peak levels at 8-10 days (Figure 1A), which were sustained for weeks (not shown). Thus, the p38MAPK response to senescence-inducing genotoxic stress differed markedly in kinetics from the response to an acute stress (e.g., LPS stimulation) (Cuenda and Rousseau, 2007) that does not induce senescence. Importantly, the kinetics of p38MAPK activation closely paralleled the kinetics with which the SASP develops (Coppe et al, 2008; Rodier et al, 2009).

\section{p38MAPK activity is required for the SASP}

p38MAPK activation during senescence was inhibited by the small molecule SB203580 (SB). SB displaces ATP from the p38MAPK ATP-binding pocket (Young et al, 1997), thereby preventing p38MAPK from phosphorylating its targets without preventing p38MAPK phosphorylation itself. As determined by Hsp27-P levels, daily treatment with $10 \mu \mathrm{M}$ $\mathrm{SB}$, which has minimal off-target effects (Cuenda et al, 1995; Wilson et al, 1997), prevented p38MAPK activation after XRA (Figure 1A).

To determine the significance of the coincident rise in p38MAPK activity and the SASP, we added SB to SEN(XRA) 
A

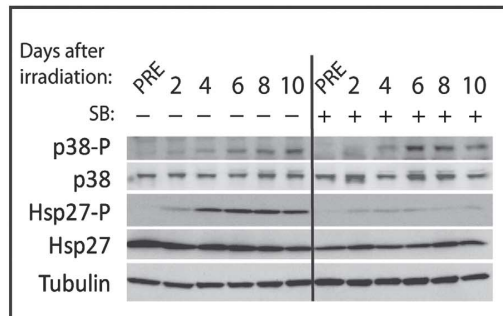

C

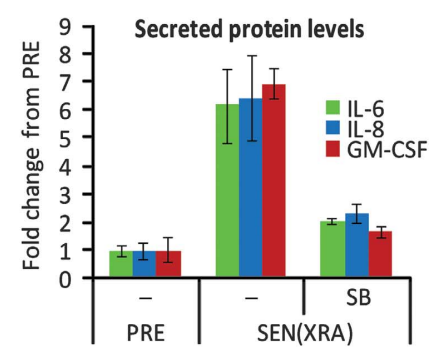

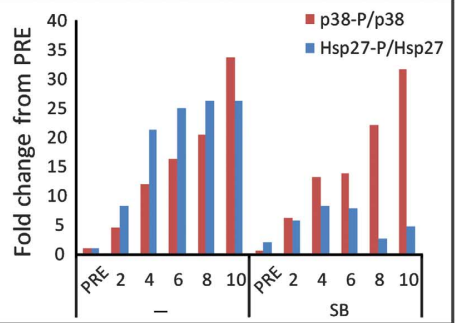

D

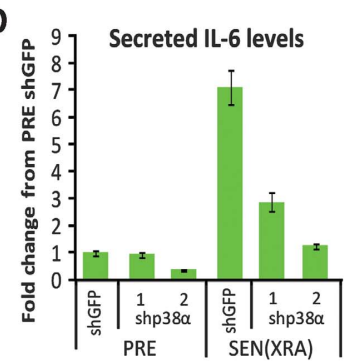

B

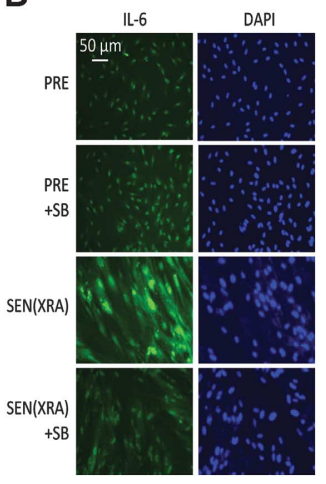

$\mathbf{F}$

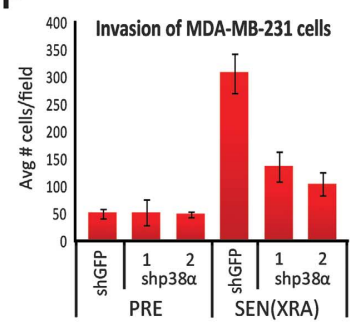

Figure 1 p38MAPK is activated with slow kinetics during genotoxic stress-induced senescence and is required for the SASP. (A) p38MAPK phosphorylation increases during DNA damage-induced senescence. Cells were irradiated and whole cell lysates collected at the indicated days thereafter. + : p38MAPK was continuously inhibited by $10 \mu \mathrm{M}$ SB203580 (SB) beginning $48 \mathrm{~h}$ before irradiation. Left: western blot analysis of indicated proteins. Right: western blot quantitation, normalized to PRE levels. p38-P, phosphorylated p38MAPK; Hsp27-P, phosphorylated heat shock protein 27. (B) p38MAPK inhibition decreases intracellular IL-6 in SEN(XRA) cells. PRE and SEN(XRA) cells immunostained for IL-6. + SB: p38MAPK was inhibited by SB023580 (SB) for $48 \mathrm{~h}$ before fixation. (C) p38MAPK inhibition decreases IL-6, IL-8, and GM-CSF secreted by SEN(XRA) cells. Conditioned media (CM) were collected from PRE and SEN(XRA) cells and analysed by ELISA. + SB: p38MAPK was inhibited by SB023580 (SB) for $48 \mathrm{~h}$ before CM collection. (D) p38MAPK $\alpha$ depletion decreases secreted IL-6. Cells were infected with lentivirus expressing either of two shRNAs against p38MAPK $\alpha(\operatorname{shp} 38 \alpha$ ) or a control shRNA (shGFP) and selected. Cells were irradiated and allowed to senesce (SEN(XRA)). CM were analysed by ELISA. (E) p38MAPK inhibition suppresses the SEN(XRA) SASP. CM from PRE and SEN(XRA) cells, with (SB) or without p38MAPK inhibition, were analysed by antibody arrays. Shown are factors for which the SEN(XRA) level was significantly increased $(P<0.05)$ over PRE. For each protein, signals from all conditions were averaged to generate the baseline. Signals above baseline are yellow; signals below baseline are blue. The heat map key shows $\log _{2}$-fold changes from baseline. The hierarchical clustering relationship between sample profiles is shown graphically as a dendrogram (left). ${ }^{*}$ : Factors significantly decreased by p38MAPK inhibition $(P<0.05)$. (F) p38MAPK depletion decreases the ability of senescent cells to stimulate cancer cell invasiveness. CM from cells described in (D) were analysed for the ability to stimulate invasiveness of MDA-MB-231 breast cancer cells in a Boyden chamber invasion assay.

cells for $48 \mathrm{~h}$, then assessed IL-6, an indicator of SASP activity (Coppe et al, 2008, 2010a; Bhaumik et al, 2009; Orjalo et al, 2009) by immunostaining (intracellular levels) and enzymelinked immunoadsorbent assay (ELISA) of conditioned medium (CM) (secreted levels). Both assays showed that SB reduced IL-6 levels to near-PRE levels (Figures $1 \mathrm{~B}$ and C); ELISA showed that SB also significantly reduced secretion of the SASP components IL-8 and GM-CSF (Figure 1C) $(P<0.05)$. Further, SB significantly reduced secreted IL-6 levels in CM from SEN(XRA) WI-38, an unrelated human fibroblast strain (Supplementary Figure S1D), and replicatively senescent (SEN(REP)) HCA2 and WI-38 cells (Supplementary Figure S1E). Thus, the ability of p38MAPK inhibition to significantly reduce senescence-associated IL-6 secretion was not confined to XRA-induced senescence or a single cell strain. These findings suggest that p38MAPK activation is necessary for secretion of at least some SASP components.

Although SB is a well-characterized p38MAPK inhibitor, one report showed it can partially inhibit protein kinase B at the concentration used here $(10 \mu \mathrm{M})$ (Lali et al, 2000), and it inhibits both the $\alpha$ and $\beta$ p38MAPK isoforms (Enslen et al, 1998). To determine whether the effect of SB on SASP components was p38MAPK specific, and to identify the isoform that regulates the SASP, we depleted cells of p38MAPK $\alpha$, the most abundant isoform, by RNA interference (RNAi). Using lentiviruses, we expressed in SEN(XRA) cells either of two unrelated short hairpin (sh) RNAs that specifically target p38MAPK $\alpha$ (Supplementary Figure S1F). We then assayed IL-6 levels in CM from cells expressing control (shGFP) or p38MAPK $\alpha$-specific $(\operatorname{shp} 38 \alpha)$ shRNAs. Both shp38 $\alpha$ shRNAs significantly decreased secreted IL-6 levels in SEN(XRA) cells (Figure $1 \mathrm{D} ; P<0.01$ ). A p38MAPK $\beta$ shRNA did not reduce secreted IL-6 levels (not shown). These data confirm that p38MAPK is essential for induction of the SASP, and identify p38MAPK $\alpha$ as the major functional isoform.

The SASP is a complex network comprising $>40$ proteins (Coppe et al, 2008, 2010b). To determine which SASP factors are p38MAPK regulated, we analysed CM from PRE and SEN(XRA) cells, with or without p38MAPK inhibition, using arrays containing antibodies against 120 secreted proteins. This analysis identified 37 proteins that were significantly upregulated in SEN(XRA) cells relative to PRE (Figure 1E). Most of these proteins $(68 \%, 25 / 37)$ declined significantly $(P<0.05)$ following p38MAPK inhibition (SEN $(\mathrm{XRA})+\mathrm{SB}$ ) (Figure 1E, asterisks; Supplementary Figure S1G); the remaining SASP proteins exhibited non-significant decreases (Figure 1E). The 
p38MAPK-regulated proteins included cytokines, chemokines, growth factors, shed ligands and, importantly, 9 of the 10 most robustly secreted SASP proteins. Hierarchical clustering (Eisen et al, 1998) of the array results showed that SEN(XRA) cells treated with SB had an SASP profile that more closely resembled PRE cells than untreated SEN(XRA) cells (Figure 1E). p38MAPK inhibition slightly increased secreted levels of a few proteins, but not significantly (Supplementary Figure $\mathrm{S} 1 \mathrm{H}$ ). The SASP also includes several MMPs, most prominently MMP1 and MMP3 (Coppe et al, 2010b). p38MAPK inhibition had little effect on secreted MMP1 or MMP3 levels; even when we started p38MAPK inhibition before XRA and continued until sample collection, only MMP3 declined; MMP1 was not affected (Supplementary Figure S1I). Thus, p38MAPK is a less potent regulator of SASP MMPs, but a strong positive regulator of many SASP chemokines, cytokines, and growth factors.

\section{p38MAPK inhibition mitigates a paracrine effect of senescent cells}

CM from senescent cells stimulate the ability of cancer cells to invade a basement membrane (Coppe et al, 2008). To determine whether p38MAPK inhibition mitigates this effect, we measured the ability of CM from PRE or SEN(XRA) cells expressing either a control (shGFP) or p38MAPK $\alpha(\operatorname{shp} 38 \alpha)$ shRNA to stimulate the invasiveness of MDA-MB-231 human breast cancer cells. SEN(XRA) CM stimulated $\sim 6$-fold more invasion than PRE CM (Figure $1 F, P<0.001$ ), as expected. p38MAPK depletion markedly reduced this stimulatory activity (Figure $1 \mathrm{~F}, P<0.001$ ), indicating that p38MAPK inhibition can mitigate an important biological consequence of the SASP.

\section{p38MAPK inhibition mitigates the SASP induced by oncogenic RAS expression}

Senescence can be induced by certain oncogenes, including the oncogenic form of H-RAS $\left(\mathrm{RAS}^{\mathrm{V} 12}\right.$ ) (Serrano et al, 1997). As reported (Di Micco et al, 2006), oncogenic $\mathrm{H}^{-R A S}{ }^{\mathrm{V} 12}$ expression caused hyperproliferation for several days, resulting in DNA damage and ultimately a senescence growth arrest 8-10 days later (SEN(RAS)) (not shown). Like SEN(XRA) cells, and as reported (Wang et al, 2002; Deng et al, 2004), SEN(RAS) cells showed increased levels of activated (phosphorylated) p38MAPK (Figure 2A). Also as reported (Coppe et al, 2008), SEN(RAS) cells expressed an amplified SASP, secreting several proteins at significantly higher levels than those secreted by SEN(XRA) cells and several factors not present in the SEN(XRA) SASP. In HCA2 cells, the SEN(RAS) SASP included 83 proteins (Figure 2B; Supplementary Figure S2A). p38MAPK inhibition $($ SEN $(R A S+S B))$ significantly reduced $(P<0.05)$ secreted levels of $78 \%(65 / 83)$ of these proteins (Figure 2B; Supplementary Figure S2A, asterisks), including 9 of the 10 most robustly secreted SASP proteins. The remaining SASP proteins were non-significantly reduced (Supplementary Figure S2A). p38MAPK inhibition also significantly reduced MMP1 and MMP3 levels in SEN(RAS) cells, although to a lesser extent than most cytokines and chemokines (Supplementary Figure S2B). The SASP proteins affected by p38MAPK inhibition in SEN(RAS) cells overlapped with many of those affected by p38MAPK inhibition in SEN(XRA) cells: of the 23 factors upregulated in both SEN(XRA) and
SEN(RAS), 78\% (19/23) were significantly decreased by p38MAPK inhibition in both cases (Supplementary Figure S2C). Thus, a majority of the SASP factors induced by both genotoxic stress (XRA) and oncogene activation (RAS) depends on p38MAPK activity.

p53 restrains the SASP by restraining p38MAPK activity p53 restrains the SASP via an unknown mechanism (Coppe et al, 2008). To determine the relationship between p53 and p38MAPK during development of the SASP, we inactivated p53 using retrovirally-delivered GSE22, a peptide that prevents p53 tetramerization and thus p53 transcriptional activity (Ossovskaya et al, 1996). Because p53 monomers are not rapidly degraded, GSE22 activity can be monitored by the accumulation of p53 protein (Figure 2C). We induced p53-deficient cells to senesce with XRA (SEN(XRA) + GSE), and compared phosphorylated p38MAPK levels with those in SEN(XRA) and SEN(RAS) cells.

Activated p38MAPK levels were highest in SEN(XRA) + GSE cells, followed by SEN(RAS) and SEN(XRA) (Figure 2C). The relative levels of p38MAPK phosphorylation matched the relative levels of IL-6 secretion (Figures $2 \mathrm{C}$ versus D), suggesting that p53 and RAS regulate the intensity of the SASP by regulating the level of p38MAPK activation. p53 also regulated the kinetics of SASP development by regulating the timing of p38MAPK activation. When p53 was inactivated by GSE22, p38MAPK phosphorylation occurred more rapidly after XRA compared with cells with wild-type p53 (Figure 2E). To determine whether this increase in p38MAPK activity was responsible for the amplified SASP in p53deficient cells, we inhibited p38MAPK with SB. The amplified levels of IL-6, IL-8, and GM-CSF were almost completely suppressed by p38MAPK inhibition (Figure 2F). We obtained similar results when we depleted cells of p53 by RNAi (Supplementary Figures S2D and E). We conclude that p53 restrains p38MAPK activity after senescence induction, thereby restraining the SASP.

\section{p38MAPK activity is sufficient to induce an SASP}

To study the effect of constitutive p38MAPK activation, we infected PRE cells with a constitutively active mutant (MKK6EE) of MAP kinase kinase 6 (MKK6), which directly phosphorylates p38MAPK. As expected, MKK6EE expression caused phosphorylation of endogenous p38MAPK (Figure 3A). Moreover, cells ceased proliferation several days later (Figure 3B). This growth arrest was accompanied by increased SA- $\beta$ gal activity (Supplementary Figure S3A), decreased BrdU incorporation (Supplementary Figure S3B), and a senescent morphology (Supplementary Figure S3C). These responses were prevented by p38MAPK inhibition.

To determine whether constitutive p38MAPK activity (due to MKK6EE expression) is sufficient to induce an SASP, we used antibody arrays and identified 19 factors that were significantly upregulated in MKK6EE-expressing cells relative to PRE controls (Figure 3C). p38MAPK inhibition (MKK6EE $+\mathrm{SB}$ ) significantly reduced the secreted levels of most of these proteins (84\%, 16/19) (Figure 3C, asterisks); the remaining proteins were non-significantly reduced (Figure 3C). Notably, 7 of the 10 most upregulated factors in SEN(XRA) cells, and 9 of the 10 most upregulated factors in SEN(RAS) cells, increased significantly upon MKK6EE expression (Figure 3D). We validated the array results by 
A

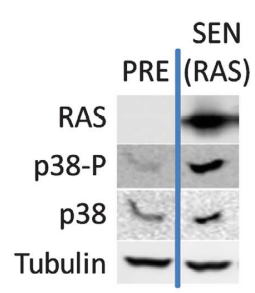

B
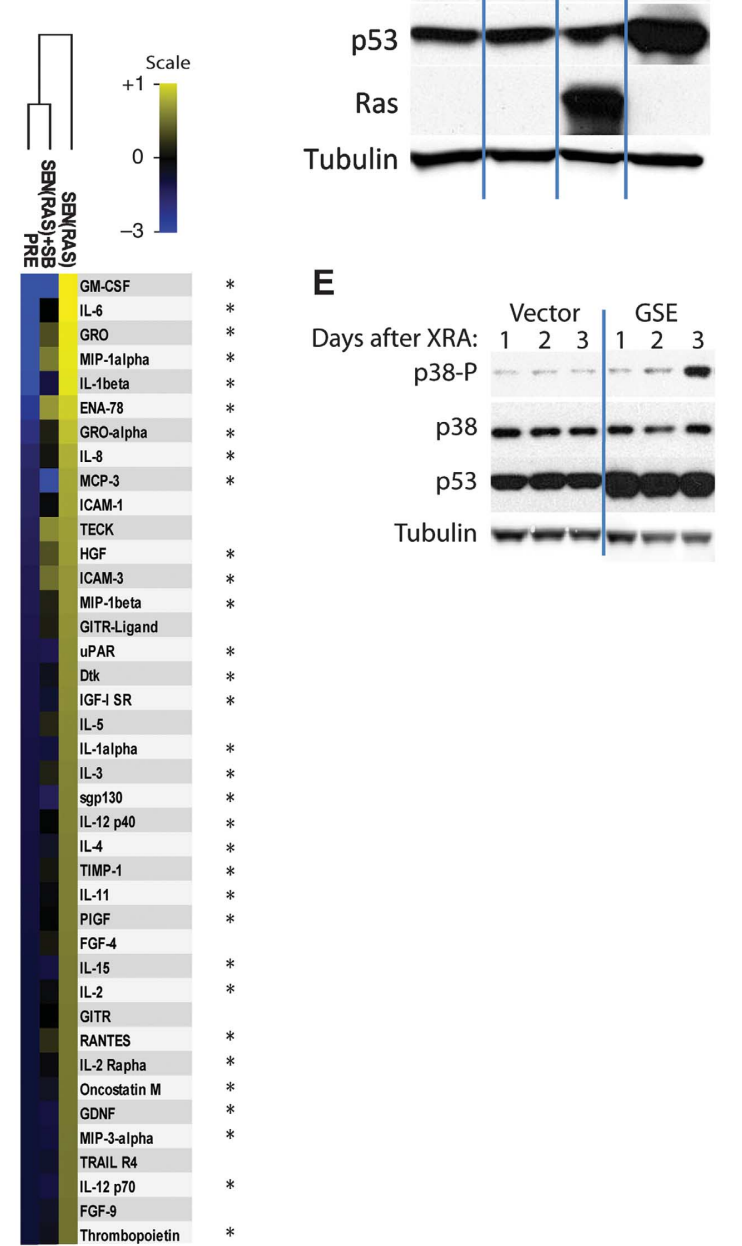

E

C

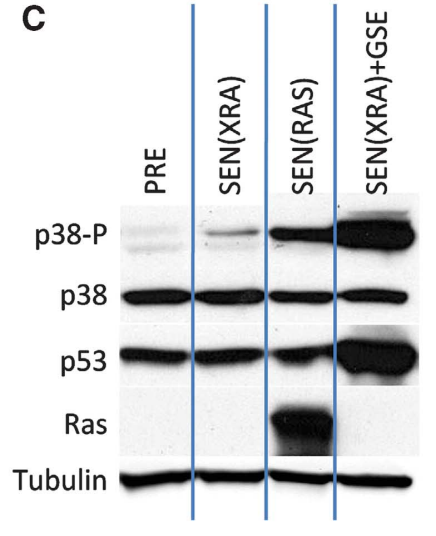

Vector GSE

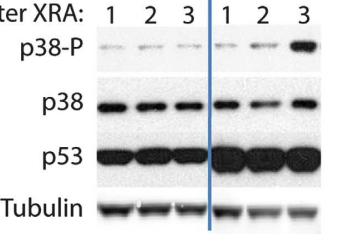

D

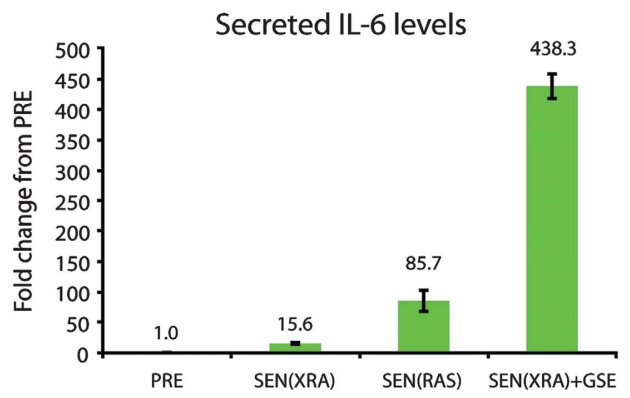

F Secreted protein levels

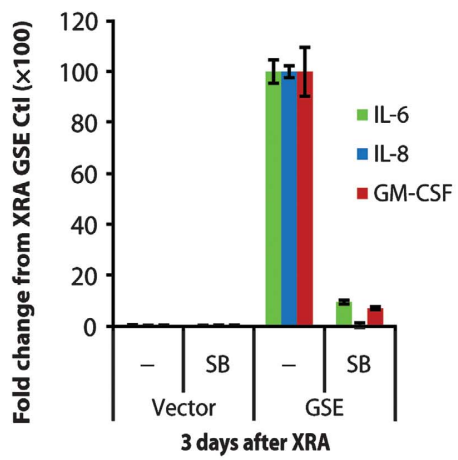

Figure 2 p38MAPK drives the amplified SASPs induced by RAS or p53 inactivation. (A) p38MAPK is phosphorylated during RAS ${ }^{\mathrm{V} 12}$-induced senescence. PRE cells were infected with lentivirus expressing oncogenic RAS ${ }^{\mathrm{V} 12}$, selected, and allowed to senesce (SEN(RAS)) for 10 days. Whole cell lysates were then collected and analysed by western blot. Presenescent controls (PRE) were infected with insertless vector. (B) p38MAPK inhibition suppresses the SEN(RAS) SASP. Cells were infected as described in (A). CM from PRE and SEN(RAS) cells were analysed by antibody arrays. + SB: p38MAPK was inhibited by SB203580 for $48 \mathrm{~h}$ before CM collection. Shown are the top 40 factors for which the SEN(RAS) level was significantly increased over PRE. PRE and SEN(RAS) values were averaged to generate the baseline. Heat map and dendrogram were generated as in Figure 1E. *: Factors significantly decreased by p38MAPK inhibition. (C) Amplified p38MAPK phosphorylation in SEN(RAS) cells and SEN(XRA) cells lacking functional p53 (SEN(XRA) + GSE). Cells were infected with lentivirus expressing GSE22 (GSE) or an insertless vector, selected, then irradiated (XRA) or infected with lentivirus expressing oncogenic RAS ${ }^{\text {12 }}$ (RAS) and allowed to senesce. Whole cell lysates were analysed by western blot. (D) SEN(RAS) and SEN(XRA) cells lacking functional p53 secrete amplified IL-6 levels. Cells were treated as in (C), then CM were collected and analysed by ELISA. (E) p53 inactivation accelerates p38MAPK phosphorylation after XRA. Cells were infected with lentivirus lacking insert (Vector) or expressing GSE22 (GSE), selected, and irradiated. Whole cell lysates were collected at specified time points and analysed by western blot. (F) GSE-amplified levels of IL-6, IL-8, and GM-CSF are p38MAPK dependent. Cells were infected as in (E) and irradiated. CM were collected 3 days later and analysed by ELISA. SB: p38MAPK was inhibited by SB203580 for $48 \mathrm{~h}$ before CM collection.

ELISA measurements of secreted IL-6 (Supplementary Figure S3D). Constitutive p38MAPK activity was also sufficient to induce MMP1 and MMP3, although to a lesser extent than it induced the cytokines and chemokines (Supplementary Figure S3E). Together, these data show that high-level constitutive p38MAPK activity is sufficient to induce a robust SASP that resembles the SEN(XRA) and SEN(RAS) SASPs, in addition to inducing growth arrest, SA- $\beta$ gal activity, and a senescent morphology.

p38MAPK regulates the SASP independent of the DDR The DDR is required for expression of a subset of SASP proteins, including IL-6 and IL-8 (Rodier et al, 2009). To determine whether p38MAPK inhibition decreases the 

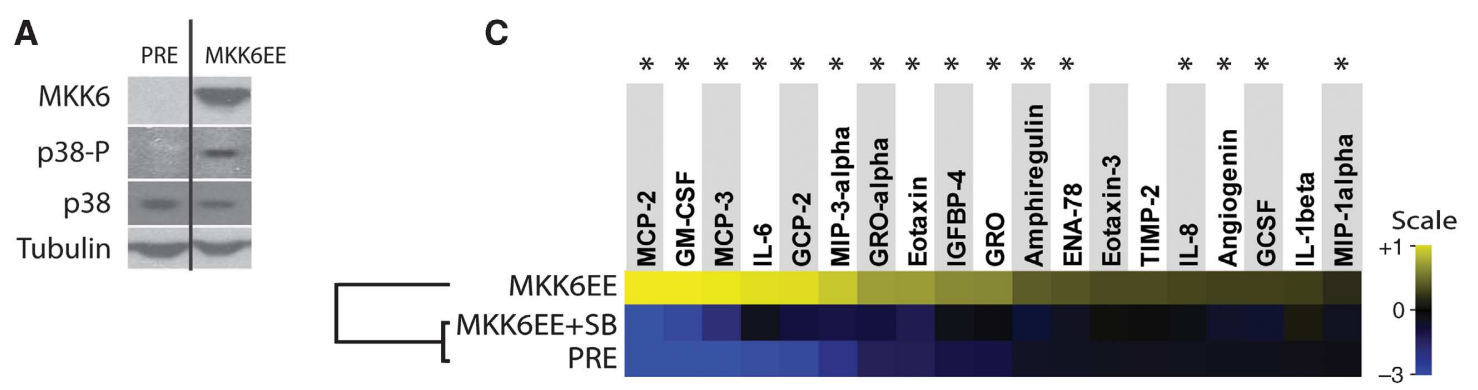

B

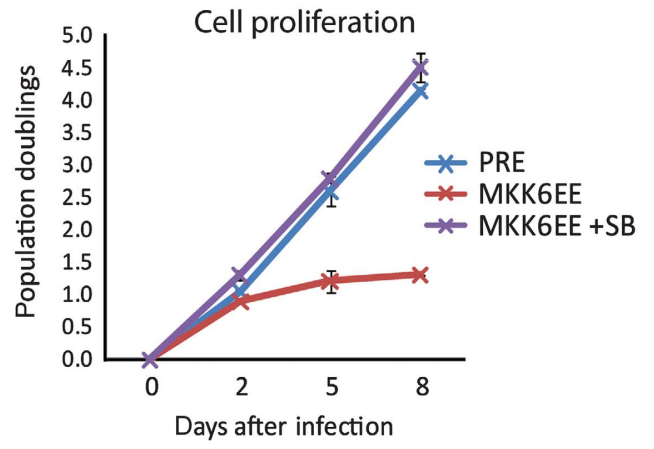

D

\begin{tabular}{|l|c|}
\hline $\begin{array}{c}\text { SEN(XRA) } \\
\text { Factor }\end{array}$ & $\begin{array}{c}\text { MKK6EE } \\
\text {-induced }\end{array}$ \\
\hline GRO & + \\
\hline IL-6 & + \\
\hline IL-8 & + \\
\hline MCP-2 & + \\
\hline MCP-3 & + \\
\hline GCP-2 & + \\
\hline MCP-1 & \\
\hline GM-CSF & + \\
\hline IGFBP-2 & \\
\hline IL-10 & \\
\hline
\end{tabular}

\begin{tabular}{|l|c|}
\hline $\begin{array}{c}\text { SEN(RAS) } \\
\text { Factor }\end{array}$ & $\begin{array}{c}\text { MKK6EE } \\
\text {-induced }\end{array}$ \\
\hline GM-CSF & + \\
\hline IL-6 & + \\
\hline GRO & + \\
\hline MIP-1alpha & + \\
\hline IL-1beta & + \\
\hline ENA-78 & + \\
\hline GRO-alpha & + \\
\hline RANTES & \\
\hline IL-8 & + \\
\hline MCP-3 & + \\
\hline
\end{tabular}

Figure 3 Constitutive p38MAPK activation is sufficient to induce an SASP. (A) PRE cells were infected with lentivirus expressing a constitutively active MAP kinase kinase 6 mutant (MKK6EE). Whole cell lysates were collected 8 days after infection and analysed by western blot. Presenescent controls (PRE) were infected with insertless vector. (B) MKK6EE induces p38MAPK-dependent growth arrest. Cells were infected as in (A) and counted at the indicated intervals thereafter. + SB: p38MAPK was continuously inhibited by SB203580 beginning $48 \mathrm{~h}$ before infection. (C) MKK6EE induces an SASP. Cells were infected as described in (A); CM were collected 8 days after infection. + SB: p38MAPK was inhibited by SB203580 for $48 \mathrm{~h}$ before CM collection. Secreted proteins were analysed using antibody arrays. Shown are proteins for which the MKK6EE level was significantly increased $(P<0.05)$ over PRE. PRE and MKK6EE values were averaged to generate the baseline. Heat map and dendrogram were generated as in Figure 1E. *: Factors significantly decreased by p38MAPK inhibition $(P<0.05)$. $(\mathbf{D})$ The MKK6EE SASP resembles SEN(XRA) and SEN(RAS) SASPs. Shown are the 10 most upregulated factors in the SEN(XRA) and SEN(RAS) SASPs. + : Factors significantly increased by MKK6EE expression.

SASP by inhibiting the DDR, we induced senescence by XRA and measured the activities of several DDR proteins with or without p38MAPK inhibition (SB). p38MAPK inhibition had no effect on the rapid (within $2 \mathrm{~h}$ ) phosphorylation of ATM, CHK2, or p53(Ser15), nor on the transient stabilization of p53 and expression of p21 after XRA (Figure 4A). p38MAPK inhibition also did not affect the low-level activation of these DDR proteins that persists after XRA ( $>2$ days) (Rodier et al, 2009, 2011) (Figure 4A). Thus, p38MAPK inhibition does not suppress the SASP by suppressing the DDR.

In the presence of existing DNA damage, p38MAPK can replenish short-lived DNA damage foci via an ROS feedback loop (Passos et al, 2010). Supporting those findings, though p38MAPK inhibition had no effect on the formation or resolution of 53BP1 foci during the first 4 days after XRA, it slightly decreased foci number 6-8 days after XRA (Supplementary Figure S4A, $P<0.01$ ) and in replicatively senescent cells (Supplementary Figure S4B). However, in contrast to XRA, REP, and RAS, constitutive p38MAPK activity (MKK6EE expression) did not induce DNA damage foci in most cells: most SEN(RAS), SEN(XRA), and (SEN(REP) cells harboured $\geqslant 353 \mathrm{BP} 1$ foci per nucleus, but most cells induced to senesce by MKK6EE harboured $<353 \mathrm{BP} 1$ foci and were not significantly different from PRE cells (Figure $4 \mathrm{~B}, P>0.05$ ). There was a slight increase in the percentage of MKK6EE-induced senescent cells with $>10$ 53BP1 foci/nucleus, but these cells accounted for only $\sim 3 \%$ of the total (Supplementary
Figure S4C). Further, cells made senescent by MKK6EE expression did not exhibit an increase in global ATM or CHK2 phosphorylation (Figure 4C). MKK6EE expression did not increase global DNA damage signalling at any time after infection, as measured by p53(Ser15) phosphorylation, unlike RAS expression (Supplementary Figure S4D). Finally, though depletion of ATM or CHK2 by RNAi reduced IL-6, IL-8, and GM-CSF secretion by SEN(XRA) and SEN(RAS) cells, as described (Rodier et al, 2009), $>90 \%$ depletion of these DDR factors (Supplementary Figure S4E) had no effect on IL-6, IL-8, or GM-CSF secretion induced by MKK6EE (Figure 4D, $P>0.05$ ). Thus, p38MAPK does not regulate the SASP by modulating the DDR. Conversely, the DDR does not regulate the SASP by modulating p38MAPK activity. Neither ATM nor CHK2 depletion suppressed p38MAPK phosphorylation in SEN(XRA) (Figure 4E, top) or SEN(RAS) (Figure 4E, bottom) cells. Together, these findings indicate that p38MAPK activity uniquely regulates the SASP independently of the DDR.

If p38MAPK is sufficient to induce an SASP and also independent of the DDR, how can the DDR be required for the SEN(XRA) and SEN(RAS) SASPs? MKK6EE induces activation of endogenous p38MAPK to a much greater extent than XRA or RAS. To determine whether a lower level of p38MAPK activation is sufficient to induce the SASP, we blunted p38MAPK activity in MKK6EE cells with varying doses of SB203580 and measured downstream signalling via 
A
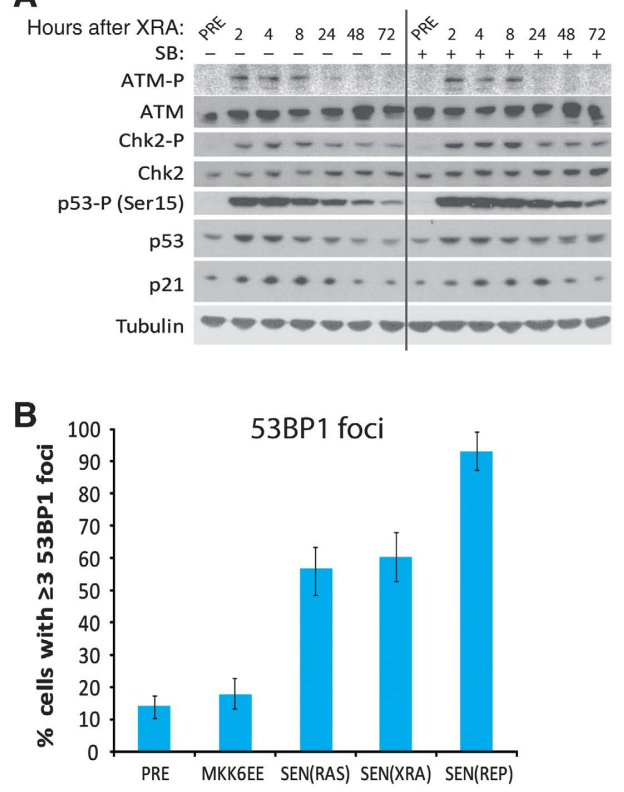

C

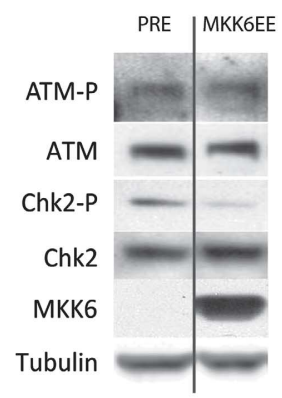

D
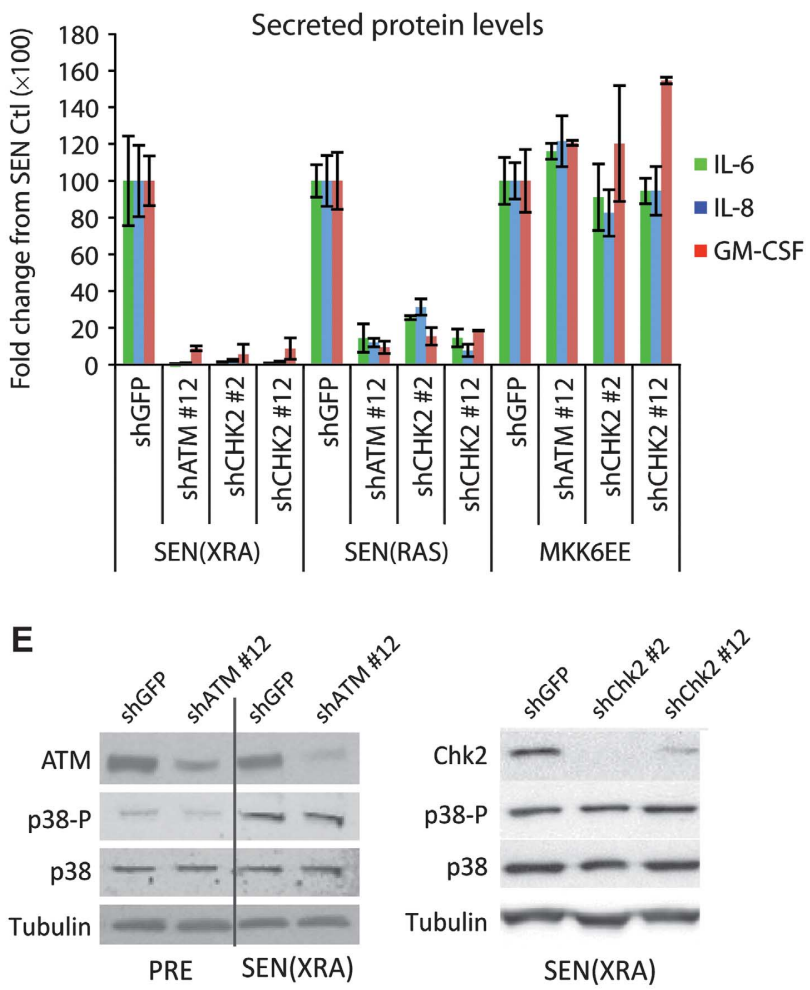

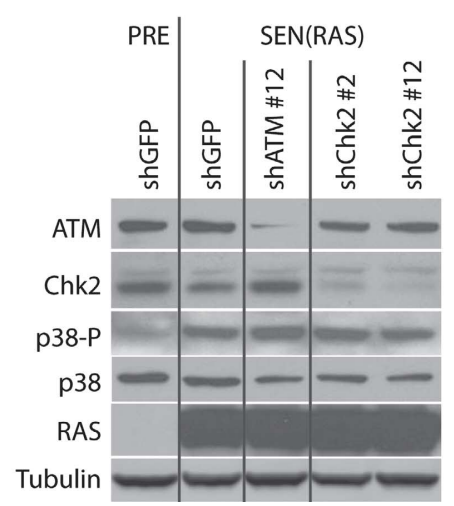

Figure 4 p38MAPK induces an SASP independent of the DNA damage response. (A) p38MAPK inhibition does not prevent the DDR. Whole cell lysates were collected at specified intervals after irradiation and analysed by western blot. Where indicated, p38MAPK was continuously inhibited by SB203580 (+) beginning 48 h before irradiation. ATM-P, Ser 1981 phosphorylated ATM; CHK2-P, Thr 68 phosphorylated CHK2. (B) Constitutive p38MAPK activation does not induce 53BP1 foci. Cells were fixed 8 days after MKK6EE expression (MKK6EE), 10 days after RAS expression (SEN(RAS)), 8 days after irradiation (SEN(XRA)), or after replicative senescence (69 population doublings) (SEN(REP)) and immunostained for 53BP1. Foci were quantified using CellProfiler to count cells with $\geqslant 353 \mathrm{BP} 1$ foci per nucleus. Error bars indicate $95 \%$ confidence interval. (C) Constitutive p38MAPK activation does not induce a DDR. Whole cell lysate was collected 8 days after MKK6EE infection and analysed by western blot. Presenescent controls (PRE) were infected with insertless vector. ATM-P, Ser 1981 phosphorylated ATM; CHK2-P, Thr 68 phosphorylated CHK2. (D) Neither ATM nor CHK2 depletion prevents the SASP induced by constitutive p38MAPK activation. PRE cells were irradiated (SEN(XRA)), infected with RAS lentivirus (SEN(RAS)), or infected with MKK6EE lentivirus (MKK6EE). Simultaneously, cells were infected with lentivirus expressing shRNAs against ATM (shATM \#12), CHK2 (shChk2 \#2, shChk2 \#12), or GFP (shGFP; control) and selected. CM from 8 days after infection/irradiation were analysed by ELISA. (E) ATM or CHK2 depletion does not prevent p38MAPK phosphorylation at senescence. Top: cells were irradiated; 6 days later cells were infected with lentivirus expressing shRNAs against ATM (shATM \#12), CHK2 (shChk2 \#2, shChk2 \#12), or GFP (shGFP; control) and selected. Bottom: cells were simultaneously infected with RAS lentivirus, lentivirus expressing an shRNA against ATM (shATM \#12), CHK2 (shChk2 \#2, shChk2 \#12), or GFP (shGFP; control) and selected. Whole cell lysates from 8 days after irradiation/infection were analysed by western blot. p38-P: phosphorylated p38.

Hsp27 phosphorylation. p38MAPK was sufficient to induce a substantial increase in IL-6 only when active at a higher level than that seen in SEN(XRA) cells (Supplementary Figure S4F). When p38MAPK signalling was inhibited to a level that matched SEN(XRA) cells (as measured by Hsp27-P), it was not sufficient to induce IL-6 to SEN(XRA) levels (Supplementary Figure S4G). Thus, though the level of p38MAPK signalling in MKK6EE cells is sufficient to induce an SASP without the DDR, the level of p38MAPK signalling in other types of senescence is not sufficient, and therefore requires cooperation with the DDR in order to induce the SASP. 


\section{p38MAPK inhibition suppresses SASP component mRNA levels}

Many SASP factors are upregulated at the level of mRNA abundance (Coppe et al, 2008, 2010b). To understand the mechanism by which p38MAPK regulates the SASP, we used quantitative RT-PCR to determine mRNA levels of six SASP factors (GM-CSF, IL-6, IL-8, GRO $\alpha$, MCP-2, and IL- $1 \alpha$ ) that declined significantly upon p38MAPK inhibition. For all six factors, p38MAPK inhibition (SB) markedly decreased mRNA abundance in SEN(XRA) cells (Figure 5A). We obtained similar results in another cell strain (Supplementary Figure S5A). For GM-CSF, IL-6, and IL-8, the decrease in mRNA abundance matched the decrease in secreted protein level (Supplementary Figure S5B). In addition, constitutive p38MAPK activation was sufficient to induce SASP mRNAs, as determined by IL-6 and IL-8 mRNA levels upon MKK6EE expression (Supplementary Figure S5C). While these results do not rule out the possibility that p38MAPK stimulates the SASP by influencing other processes (e.g., translation and secretion), the data suggest that p38MAPK induces the SASP primarily by increasing mRNA abundance.

\section{p38MAPK controls NF-кB activity in senescent cells}

Because p38MAPK is known to regulate the activity of multiple transcription factors (TFs) depending on context (Zarubin and Han, 2005), we examined the promoters of p38MAPKinduced factors (Figure 3D) for overrepresented transcription factor-binding sites (TFBS). We interrogated $200 \mathrm{bp}$ upstream of each transcriptional start site using the $243 \mathrm{TF}$ weight matrices in the TRANSFAC database. NF-кB-binding motifs were most statistically overrepresented (Figure 5B). Activated $\mathrm{NF}-\kappa \mathrm{B}$ is enriched at the IL-8 and GRO $\gamma$ promoters following MEK-induced senescence (Acosta et al, 2008). We therefore asked whether NF- $\mathrm{B}$ activity increases during multiple types of senescence, and whether the increase is p38MAPK dependent.

Inactive $\mathrm{NF}-\kappa \mathrm{B}$ dimers are sequestered in the cytoplasm by the inhibitor I $\kappa \mathrm{B}$. NF- $\kappa \mathrm{B}$ activating signals cause I $\mathrm{B}$ degrada-

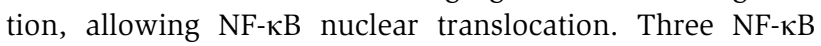
family members (RelA, RelB, and C-Rel) have DNA binding and transactivation domains, but RelA is most strongly associated with inflammatory cytokine gene transcription (Karin, 2006; Perkins, 2007). RelA was mostly cytoplasmic in PRE cells, but noticeably more nuclear in SEN(XRA) cells (Figure 5C). Further, NF- $\mathrm{B}$ DNA-binding activity increased $\sim 5$ fold in SEN(REP), SEN(RAS), and SEN(XRA) cells (two strains) (Supplementary Figures S5D and E, $P<0.001$ ). Constitutive p38MAPK activity (MKK6EE expression) was sufficient to induce this activity (Supplementary Figure S5D). Importantly, following XRA, NF- $\mathrm{B}$ DNA-binding activity increased slowly with kinetics that followed p38MAPK activation (Supplementary Figure S5F): NF-кB DNA-binding activity remained near-PRE levels for $8 \mathrm{~h}$ after XRA, began to rise $24 \mathrm{~h}$ after XRA and reached maximal levels 8-10 days later (Supplementary Figure S5F). Additionally, chromatin immunoprecipitation showed increased RelA binding to the promoters of three SASP genes (Figure 5D, $P<0.01$ ). A lentiviral-delivered luciferase reporter driven by an NF- $\kappa B$ responsive promoter showed that NF- $\kappa \mathrm{B}$ transcriptional activity was > 30-fold higher in SEN(XRA) compared with PRE cells (Figure 5E), and p38MAPK inhibition (SB) significantly decreased reporter activity $(P<0.001)$. We conclude that $\mathrm{NF}-\kappa \mathrm{B}$ activity is regulated by p38MAPK during senescence.

Depletion of ATM also decreased NF- $\kappa B$ reporter activityto roughly the same level as p38MAPK inhibition (Figure 5E, $P<0.001$ ). Although increased concentrations of SB203580 had no additional effect on NF- $\kappa \mathrm{B}$ reporter output (data not shown), the combined treatment of SB203580 and ATM depletion had a synergistic effect, lowering NF- $\kappa B$ transcriptional activity to PRE control levels (Figure 5E, SB + shATM). These data reinforce the idea that ATM depletion and SB affect NF- $\kappa \mathrm{B}$ via different pathways.

\section{$N F-\kappa B$ is required for the SASP}

Because p38MAPK regulates both the SASP and NF- $\kappa B$ activity, we asked whether NF- $\kappa B$ is required for the SASP. We expressed either of two unrelated shRNAs against RelA, both of which efficiently decreased RelA levels without substantially affecting RelB or C-Rel levels (Supplementary Figure S5G). RelA depletion significantly decreased $73 \%$ $(27 / 37)$ of the SASP proteins secreted by SEN(XRA) cells (Figure 5E, asterisks), including MMP1 and MMP3 (Supplementary Figure $\mathrm{S} 5 \mathrm{H}$ ), and non-significantly decreased the remaining SASP proteins (Figure 5F). Hierarchical clustering showed that RelA-depleted SEN(XRA) cells had an SASP profile that more closely resembled PRE cells than unmodified SEN(XRA) cells (Figure 5F). There was substantial overlap between the RelA-dependent and p38MAPK-dependent (Figure 1) SASP factors: 76\% (19/25) of p38MAPK-dependent factors were also RelA dependent (Figure 5G, Venn diagram). Of the 10 most robustly secreted SASP proteins from Figure 1, 8 were both p38MAPK- and RelA-dependent (Figure 5G, table). Using IL-6, IL-8, and GM-CSF as SASP markers, we verified the RelA dependence of the SASP for SEN(RAS) cells (Figure 5H), and two cell strains (Supplementary Figure S5I). Finally, we demonstrated that IL-6, IL-8, and GM-CSF secretion induced by MKK6EE were RelA dependent (Supplementary Figure S5J). Thus, p38MAPK acts primarily through NF- $\kappa B$ to induce the SASP.

\section{Discussion}

The SASP develops when cells experience a stress severe enough to cause a senescence response. These stresses are primarily genotoxic, leading to activation of a DDR. Persistent DDR signalling is necessary for the expression of several SASP factors (Rodier et al, 2009); depletion of DDR proteins such as ATM, NBS1, or CHK2 suppresses the expression of SASP components, including IL-6 and IL-8. However, the DDR is activated immediately after damage, whereas the SASP takes days to develop, indicating that canonical DDR signalling is not sufficient for SASP expression. Thus, there must be other, delayed molecular events that are required for development of the SASP and are regulated independently of the DDR. We show here that activation of the p38MAPK/NF- $\kappa B$ pathway is such an event.

Unlike the rapid and transient response to acute stresses such as LPS stimulation, the kinetics of p38MAPK activation after DNA damage were slow and chronic, coinciding with expression of the SASP. p38MAPK inhibition effectively collapsed the senescence-associated cytokine network, preventing the pro-invasion paracine effects of senescent cells. Further, robust p38MAPK activation, caused by MKK6EE 
A

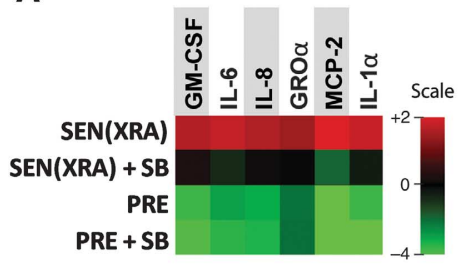

B

Overrepresented TFBS in the set of MKK6EE-induced genes

\begin{tabular}{|rl|l|c|c|}
\multicolumn{1}{r}{ Rank } & TRANSFAC Matrix ID & \multicolumn{1}{c}{ Transcription factor } & \% of sequences & P-value \\
\hline 1 & V\$NFKB_Q6 & NF-kappaB & $44 \%$ & $2.21 \mathrm{E}-10$ \\
\hline 2 & V\$CEBPA_01 & C/EBPalpha & $44 \%$ & $1.50 \mathrm{E}-09$ \\
\hline 3 & V\$CEBPB_01 & C/EBPbeta & $50 \%$ & $3.26 \mathrm{E}-09$ \\
\hline 4 & V\$NFKAPPAB65_01 & NF-kappaB (p65) & $44 \%$ & $6.37 \mathrm{E}-09$ \\
\hline 5 & V\$YY1_01 & VY1 & $50 \%$ & $7.91 \mathrm{E}-09$ \\
\hline 6 & V\$CEBP_Q2 & C/EBP & $50 \%$ & $2.94 \mathrm{E}-08$ \\
\hline 7 & V\$PBX1_01 & Pbx-1 & $50 \%$ & $8.87 \mathrm{E}-08$ \\
\hline 8 & V\$CEBP_01 & C/EBP & $56 \%$ & $1.30 \mathrm{E}-07$ \\
\hline 9 & V\$OCT1_03 & 1-Oct & $56 \%$ & $1.39 \mathrm{E}-07$ \\
\hline 10 & V\$NFKB_C & NF-kappaB & $39 \%$ & $1.41 \mathrm{E}-07$ \\
\hline
\end{tabular}

C

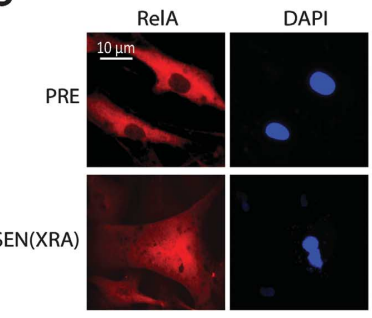

D

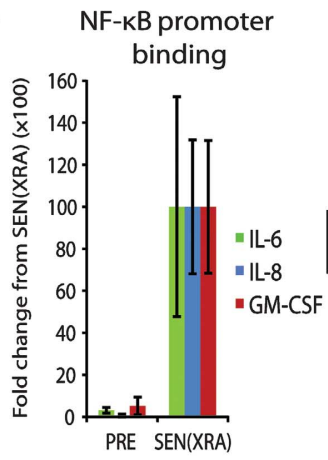

E

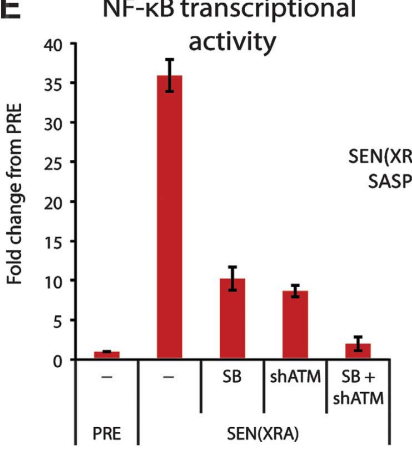

$\mathbf{F}$

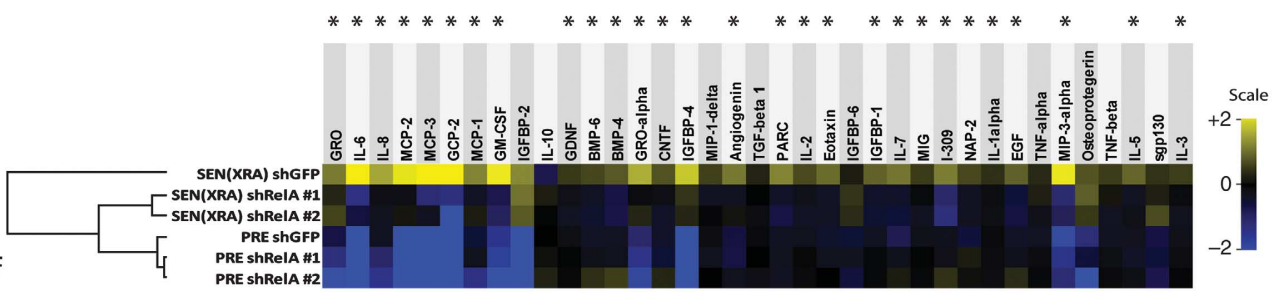

Figure 5 p38MAPK induces SASP mRNAs by increasing NF- $\kappa B$ activity. (A) p38MAPK inhibition decreases SASP mRNA levels. Total RNA was extracted from PRE and SEN(XRA) HCA2 cells; mRNA levels for indicated genes were analysed by qRT-PCR. + SB: p38MAPK was inhibited with SB203580 for $48 \mathrm{~h}$ before sample collection. For each gene, the four signals were averaged to generate the baseline. Signals above baseline are red; signals below baseline are green. The heat map key shows $\log _{2}$-fold changes from baseline. p38MAPK inhibition significantly decreased $(P<0.05)$ mRNA levels for all genes assayed. (B) Transcription factor (TF) binding sites (BS) in MKK6EE-induced genes. Genes encoding SASP proteins upregulated by MKK6EE expression (Figure 3C) were analysed for statistically overrepresented TFBS in the $200 \mathrm{bp}$ upstream of the transcriptional start site. ' $\%$ of sequences' indicates percentage of sequences with $\geqslant 1$ binding site for each indicated weight matrix. TFBS are sorted by $P$-value. (C) RelA partially localizes to the nucleus during damage-induced senescence. PRE and SEN(XRA) cells were immunostained for RelA; representative images are shown. (D) Increased RelA binding to the promoters of SASP genes. Cells were irradiated, allowed to senesce (SEN(XRA)), and lysates were analysed by chromatin immunoprecipitation using an antibody against RelA. RelA binding to the promoters of the indicated genes is represented relative to Histone $\mathrm{H} 3$ binding and is normalized to SEN(XRA) binding for each gene. (E) p38MAPK inhibition and ATM depletion reduce NF- $\mathrm{BB}$ transcriptional activity in senescent cells. Cells were infected with lentivirus expressing an NF- $\kappa B$ luciferase reporter construct, irradiated, and allowed to senesce (SEN(XRA)). Cells were lysed and luciferase activity was measured. SB: p38MAPK was inhibited with SB203580 for $48 \mathrm{~h}$ before lysis. shATM: cells were infected with lentivirus expressing shRNA against ATM 5 days before lysis. (F) RelA depletion suppresses the SASP of SEN(XRA) cells. Cells were infected with lentivirus expressing either of two shRNAs against RelA (shRelA) or GFP (shGFP; control), selected, irradiated, and allowed to senesce (SEN(XRA)). Secreted proteins were detected by antibody arrays as in Figure 1 . Shown are factors for which the SEN(XRA) level was significantly increased $(P<0.05)$ over PRE. For each protein, the six signals were averaged to generate the baseline. Heat map and dendrogram were generated as in Figure 1E. Asterisks indicate factors that are significantly decreased by both RelA shRNAs $(P<0.05)$. (G) Most p38MAPK-dependent SASP proteins are NF- $\kappa B$ dependent. Left: proportional Venn diagram displaying the overlap between p38MAPK-dependent factors (red), RelA-dependent factors (blue), and the SEN(XRA) SASP (yellow). In all, 76\% of p38MAPK-dependent factors are also RelA-dependent (dashed area). Right: the 10 most upregulated SEN(XRA) SASP factors from Figure 1E. + : Proteins dependent on RelA or p38MAPK. (H) SEN(RAS)-induced IL-6, IL-8, and GMCSF are RelA-dependent. Cells were infected with lentivirus expressing either of two shRNAs against RelA (shRelA) or GFP (shGFP; control) and selected. Cells were then infected with lentivirus lacking an insert (PRE) or expressing RAS ${ }^{v 12}$ and allowed to senesce (SEN(RAS)). CM were analysed by ELISA.

expression, was sufficient to induce an SASP, suggesting that p38MAPK activity is limiting for SASP development.

Of particular importance, we found that p53 restrains the SASP by restraining p38MAPK activity. When we inactivated p53 and then delivered a senescence-inducing genotoxic stress, p38MAPK was activated faster and to a higher level. The increased activation correlated with the amplified SASP that develops in the absence of p53 (Coppe et al, 2008), and 
p38MAPK inhibition reduced the amplified SASP. Given that transformed and primary cells can differ in the kinetics with which the p38MAPK/NF-kB pathway is activated (Janssens and Tschopp, 2006), our data suggest that p53 represses the p38MAPK pathway immediately after DNA damage in normal cells. This repression may allow time to repair the damage before cells commit to developing an SASP, which signals to the tissue microenvironment.

The p38MAPK pathway acted in parallel to the DDR. Inhibition of p38MAPK did not affect activation of important DDR factors such as ATM, CHK2, or p53. Additionally, constitutive p38MAPK activation, despite inducing an SASP, neither induced ATM or CHK2 activation nor increased DNA damage foci in most cells. Furthermore, ATM or CHK2 depletion had no effect on the p38MAPK-induced SASP. Thus, we show for the first time that senescence-associated pro-inflammatory cytokine secretion can occur in the absence of a DDR. Additionally, neither ATM nor CHK2 depletion altered p38MAPK phosphorylation at senescence, suggesting that p38MAPK is not downstream of the canonical DDR. p38MAPK inhibition had a small effect on the resolution of DNA damage foci in a subset of senescent cells, supporting reports that p38MAPK can replenish short-lived DNA damage foci (Passos et al, 2010). However, as the p38MAPK-induced SASP did not require ATM or CHK2, the DDR-ROS feedback loop that maintains a subset of DNA damage foci (Passos et al, 2010) does not seem to be the mechanism by which p38MAPK regulates the SASP.

$\mathrm{NF}-\kappa \mathrm{B}$ was the crucial effector of p38MAPK signalling during senescence. NF- $\kappa \mathrm{B}$-binding sites were the most statistically overrepresented TFBS in the identified set of p38MAPK-induced genes, and NF- $\mathrm{B}$ activity was increased in DNA damage-induced, oncogene-induced, and replicative senescence. Moreover, p38MAPK increased SASP factor mRNA abundance, and p38MAPK was required for senescence-induced NF- $\kappa$ B activity. ATM was also required for senescence-induced NF- $\kappa \mathrm{B}$ activity and acted synergistically with p38MAPK, supporting the independence of the DDR and p38MAPK pathways. The SASPs induced by DNA damage, RAS, or constitutive p38MAPK activity all required NF- $\kappa B$, demonstrating its role in mediating p38MAPK signalling. NF- $\kappa \mathrm{B}$ may not be the only means by which p38MAPK increases SASP gene expression-other TFBS such as $\mathrm{C} / \mathrm{EBP}$ sites were also overrepresented in p38MAPK-induced SASP genes and are indirectly regulated by p38MAPK (Cortez et al, 2007), and p38MAPK can also affect mRNA stability (Wang et al, 1999; Radtke et al, 2010). Nevertheless, the SASP network was largely dependent on NF- $\mathrm{B}$, and p38MAPK was, in turn, necessary and sufficient for NF- $\kappa$ B activity.

p38MAPK activity was sufficient for SASP activity, but only at high levels. At the level of activation caused by XRA or RAS, p38MAPK was not sufficient to activate the SASP-the DDR was also necessary. We propose a model in which the DDR and the p38MAPK pathways each post-translationally modify specific NF- $\mathrm{B}$ sites, with varying degrees of efficiency. Both p38MAPK and ATM are required for NF- $\mathrm{NB}$ activity at senescence, and NF- $\mathrm{BB}$ requires simultaneous phosphorylation on multiple sites for its activity (Karin, 2006; Perkins, 2007). We suggest that p38MAPK or its downstream target(s) have a high affinity for some sites, which become phosphorylated by the amount of p38MAPK signalling caused by XRA or RAS, but low affinity for sites normally phosphorylated by ATM or its downstream targets. Those low-affinity sites, then, are phosphorylated by p38MAPK only if p38MAPK signalling is high (e.g., due to MKK6EE expression). In SEN(XRA) and SEN(RAS) cells, p38MAPK signalling is not high enough to result in phosphorylation of the lowaffinity sites, making both p38MAPK and the DDR necessary

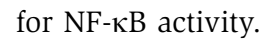

p38MAPK was crucial for the expression of many SASP cytokines and chemokines, which are largely pro-inflammatory and pro-carcinogenic (Davalos et al, 2010; Freund et al, 2010; Coppe et al, 2010a). However, p38MAPK was less important for the SASP MMPs, which may be beneficial in the short term. The MMPs have matrix-degrading and fibrolytic activity, which may limit fibrosis during wound healing (Krizhanovsky et al, 2008; Jun and Lau, 2010). The two most highly secreted MMPs are MMP1 and MMP3 (Coppe et al, 2010b). p38MAPK activation induced these MMPs, but to a lesser extent than many cytokines and chemokines. Additionally, although prolonged p38MAPK inhibition reduced MMP levels in SEN(XRA) and SEN(RAS) cells, the effect was substantially smaller than the reduction in IL-6, IL-8, GM-CSF, and other SASP factors. Thus, other MMPregulating pathways must be active at senescence. More importantly, our data suggest it may be possible to reduce some SASP factors without affecting others, potentially mitigating the deleterious effects without strongly mitigating the benefits.

p38MAPK establishes the senescence growth arrest by mediating RAS-induced expression of p16 ${ }^{\mathrm{INK} 4 \mathrm{~A}}$ and directly and indirectly phosphorylating p53 (Sun et al, 2007; Kwong et al, 2009). Neither p $16^{\mathrm{INK} 4 \mathrm{a}}$ nor p53 are required for the SASP (Coppe et al, 2008, 2010a). Thus, although the SASP can indirectly reinforce the growth arrest through an IL-6/IL-8 autocrine feedback loop (Acosta et al, 2008; Kuilman et al, 2008), the above findings and our data that p38MAPK regulates the SASP via NF- $\mathrm{B}$ suggest that p38MAPK may define a divergence point for events that activate the SASP versus the growth arrest.

Our identification of the p38MAPK/NF- $\mathrm{BB}$ pathway as a necessary and sufficient, DNA damage-independent regulator of the SASP provides new insights into how senescent cells might be a source of the chronic inflammation that is a hallmark of aging and many age-related diseases.

\section{Materials and methods}

\section{Cell culture}

Primary human fibroblasts and MDA-MB-231 cells were cultured in a $10 \% \mathrm{CO}_{2}, 3 \% \mathrm{O}_{2}$ atmosphere (Coppe et al, 2008). Unless noted otherwise, 'fibroblast' or 'cells' in the text and legends refer to HCA2 fibroblasts. Presenescent (PRE) HCA2 cells completed $<35$ population doublings and had a 24-h BrdU labeling index of $>60 \%$. Cells were made replicatively senescent (SEN(REP)) by repeated subculture (Dimri et al, 1995; Krtolica et al, 2001). For DNA damage-induced senescence (SEN(XRA)), cells were grown to confluence, exposed to $10 \mathrm{~Gy} \mathrm{X}$-ray and, unless noted otherwise, analysed 8-10 days later; PRE cells were mock irradiated. For oncogene-induced senescence (SEN(RAS)), cells were infected with lentivirus expressing RAS ${ }^{\mathrm{V} 12}$ and analysed 8-10 days after infection. Where indicated, cells were given $10 \mu \mathrm{M}$ SB203580 (Calbiochem, $559395)$ for the specified intervals with daily media changes.

\section{Vectors, viruses, and infections}

MKK6EE (provided by Dr Eisuke Nishida of Kyoto University), Genetic suppressor element 22 (GSE) (Ossovskaya et al, 1996), and 
$\mathrm{RAS}^{\mathrm{V} 12}$ were subcloned into Gateway destination vector 670-1 (Campeau et al, 2009). Insertless vector was used as a control for PRE cells. Lentiviral vectors encoding shRNAs against GFP (RHS4459), p38 $\alpha$ (TRCN0000000509, TRCN0000010051), and RelA (TRCN0000014686, TRCN0000014687) were from Open Biosystems. Lentiviral vectors encoding ATM shRNAs, CHK2 shRNAs, p53 shRNA (Rodier et al, 2009) and virus production were as described (Naldini et al, 1996; Beausejour et al, 2003). Viral titres were adjusted to infect $\sim 90 \%$ of cells. Cells were infected overnight with polybrene, allowed to recover for $48 \mathrm{~h}$, selected for $48 \mathrm{~h}$, and allowed to recover for at least another $48 \mathrm{~h}$ before use.

\section{Immunofluorescence}

Cells were fixed, permeabilized, blocked, and incubated with primary antibodies overnight at $4^{\circ} \mathrm{C}$ in PBS $+1 \%$ BSA. Primary antibodies were from R\&D Systems (IL6, AF206NA, 1:60), Novus Biologicals (53BP1, NB 100-305, 1:2000), and BD Biosciences (BrdU, 347580, 1:100). Images were quantitated using CellProfiler (Carpenter et al, 2006).

\section{Senescence-associated $\beta$-galactosidase assay}

Cells were fixed and stained for SA- $\beta$ gal using BioVision's Senescence Detection Kit (\#K320-250) for $24 \mathrm{~h}$. Staining was visualized by light microscopy; positive cells were counted manually.

\section{BrdU incorporation}

Subconfluent cells were incubated for $24 \mathrm{~h}$ with $10 \mu \mathrm{M}$ BrdU and immunostained for BrdU as described (Rodier et al, 2011).

\section{Western blot analysis}

On-plate lysis was performed with either denaturing (5\% SDS, $10 \mathrm{mM}$ Tris) or non-denaturing (Cell Lysis Buffer, Cell Signalling, 9803) buffer containing protease and phosphatase inhibitors. Primary antibodies are listed in Supplementary Table S1. Signals were quantified with LI-COR Odyssey software.

\section{ELISAs and conditioned media}

ELISA kits to detect IL-6 (D6050), IL-8 (D8000C), and GM-CSF (DGM00) were from R\&D Systems. MMP1 and MMP3 were detected with AlphaLISA kits from Perkin-Elmer (AL242C, AL284C). CM were prepared by washing with serum-free DMEM and incubating in serum-free DMEM for $24 \mathrm{~h}$. All ELISA data were normalized to cell number.

\section{Antibody arrays}

$\mathrm{CM}$ samples were diluted to equivalent cell numbers in serum-free DMEM. Antibody arrays were from Raybiotech (AAH-CYT-G1000-8). Arrays were scanned using a GenePix 4200A Professional microarray scanner at $10 \mu \mathrm{m}$ resolution. Signal intensities were quantitated using LI-COR Odyssey software, and normalized to positive controls for each sample, which were then normalized across all samples.

\section{Invasion assay}

CM were diluted to equivalent cell numbers in serum-free DMEM. Invasion assays were performed as described (Coppe et al, 2006) using Matrigel Invasion Chambers $(8 \mu \mathrm{m}$ pore, BD Biosciences 354480).

\section{Quantitative RT-PCR}

Taqman analyses were performed by the UCSF Genome Core. Samples were normalized to $\beta$-glucuronidase (GUS). Primers were from Applied Biosystems: IL-1 $\alpha$ (Hs 00174092 m1), IL-6

\section{References}

Acosta JC, O’Loghlen A, Banito A, Guijarro MV, Augert A, Raguz S, Fumagalli $M$, Da Costa $M$, Brown C, Popov N, Takatsu Y, Melamed J, d'Adda di Fagagna F, Bernard D, Hernando E, Gil J (2008) Chemokine signalling via the CXCR2 receptor reinforces senescence. Cell 133: 1006-1018

Bavik C, Coleman I, Dean JP, Knudsen B, Plymate S, Nelson PS (2006) The gene expression program of prostate fibroblast senescence modulates neoplastic epithelial cell proliferation through paracrine mechanisms. Cancer Res 66: 794-802
(Hs $00174131 \mathrm{~m} 1$ ), IL-8 (Hs $00174103 \mathrm{ml}$ ), GRO $\alpha$ (Hs 00236937 m1), GM-CSF (Hs 00929873 m1), and MCP-2 (Hs 99999026 m1).

\section{$N F$ - $\kappa$ B assays}

NF-кB DNA-binding activity in whole cell lysate was assayed using the TransAM ${ }^{\mathrm{TM}}$ NF- $\kappa \mathrm{B}$ p65 Transcription Factor Assay Kit from Active Motif (40096). To assay transcriptional activity, cells were infected with Cignal NF-кB Lentiviral Reporter (SABiosciences, CLS013L) and selected. After senescence induction, cells were lysed and assayed for luciferase using the Luciferase Assay System (Promega, E1500). All values for both assays were normalized to cell number.

\section{Chromatin immunoprecipitation}

Chromatin immunoprecipitation was performed with the MAGnify ChIP system (Invitrogen, \#49-2024) and signal was detected with Roche's UPL system. In all, $3 \mu \mathrm{g}$ of RelA antibody (Santa Cruz, \#SC-372X) or Histone H3 (Abcam, \#AB-1791) was used. Primers used: IL-6 promoter: F-cacagaagaactcagatgactgg, R-aaaaccaaagatgttc tgaactga; IL-8 promoter: F-catcagttgcaaatcgtgga, R-gaacttatgcaccctc atcttttc; GM-CSF promoter: F-cccettactggactgaggttg, R-cccactgacagtt cacatgg.

\section{Analysis of TFBS}

TFM-Explorer (Defrance and Touzet, 2006) was used to identify the top 10 statistically overrepresented partial weight matrices (PWMs) in the $200 \mathrm{bp}$ upstream of the transcription start sites of genes encoding proteins significantly induced by MKK6EE expression. We searched all vertebrate PWMs available in the TRANSFAC database using a cluster density ratio of 2.5. P-values were calculated compared with a background model incorporating all RefSeq genes (24328).

\section{Statistical analyses}

Statistical analyses of main figures are in Supplementary Table S1; statistical analyses of supplementary figures are in Supplementary Table S2. Statistical significance between distributions of signals was evaluated using a two-tailed Student's $t$-test and assumption of equal variance. Statistical significance between binary assays (i.e., positive and negative scores) was evaluated using a $\chi^{2}$-test.

\section{Supplementary data}

Supplementary data are available at The EMBO Journal Online (http://www.embojournal.org).

\section{Acknowledgements}

We thank Drs Eisuke Nishida (Kyoto University) for the pSRalphamyc-MKK6-EE vector, Pierre Desprez (California Pacific Medical Center) for critically reading the manuscript, and Shruti Waghray for technical assistance. This work was supported by grants from the National Institutes of Health (AG09909, AG017242, and AG25901, JC), a National Science Foundation Graduate Research Fellowship (AF), and a fellowship from the Larry L Hillblom Foundation (CP).

Author contributions: AF and JC conceived the project; $\mathrm{AF}$ performed most of the experiments and prepared the figures; CKP participated in the array analyses; $\mathrm{AF}, \mathrm{CKP}$, and JC participated in interpreting the data; $\mathrm{AF}$, CKP, and JC wrote the paper.

\section{Conflict of interest}

The authors declare that they have no conflict of interest.
Beausejour CM, Krtolica A, Galimi F, Narita M, Lowe SW, Yaswen P, Campisi J (2003) Reversal of human cellular senescence: roles of the p53 and p16 pathways. EMBO J 22: 4212-4222

Beyaert R, Cuenda A, Vanden Berghe W, Plaisance S, Lee JC, Haegeman G, Cohen P, Fiers W (1996) The p38/RK mitogenactivated protein kinase pathway regulates interleukin- 6 synthesis response to tumor necrosis factor. EMBO J 15: 1914-1923 Bhaumik D, Scott G, Schokrpur S, Patil CK, Orjalo AV, Rodier F, Lithgow G, Campisi J (2009) MicroRNAs miR-146a/b negatively 
modulate the senescence-associated inflammatory mediators IL-6 and IL-8. Aging 1: 402-411

Campeau E, Ruhl VE, Rodier F, Smith CL, Rahmberg BL, Fuss JO, Campisi J, Yaswen P, Cooper PK, Kaufman PD (2009) A versatile viral system for expression and depletion of proteins in mammalian cells. PLoS One 4: e6529

Campisi J, d'Adda di Fagagna F (2007) Cellular senescence: when bad things happen to good cells. Nat Rev Mol Cell Biol 8: 729-740 Carpenter AE, Jones TR, Lamprecht MR, Clarke C, Kang IH, Friman $\mathrm{O}$, Guertin DA, Chang JH, Lindquist RA, Moffat J, Golland P, Sabatini DM (2006) CellProfiler: image analysis software for identifying and quantifying cell phenotypes. Genome Biol 7: R100

Ciccia A, Elledge SJ (2010) The DNA damage response: making it safe to play with knives. Mol Cell 40: 179-204

Collins CJ, Sedivy JM (2003) Involvement of the INK4a/Arf gene locus in senescence. Aging Cell 2: 145-150

Coppe JP, Desprez PY, Krtolica A, Campisi J (2010a) The senescence-associated secretory phenotype: the dark side of tumor suppression. Annu Rev Pathol 5: 99-118

Coppe JP, Kauser K, Campisi J, Beausejour CM (2006) Secretion of vascular endothelial growth factor by primary human fibroblasts at senescence. J Biol Chem 281: 29568-29574

Coppe JP, Patil CK, Rodier F, Krtolica A, Beausejour CM, Parrinello S, Hodgson JG, Chin K, Desprez PY, Campisi J (2010b) A human-like senescence-associated secretory phenotype is conserved in mouse cells dependent on physiological oxygen. PLoS One 5: e9188

Coppe JP, Patil CK, Rodier F, Sun Y, Munoz DP, Goldstein J, Nelson PS, Desprez PY, Campisi J (2008) Senescence-associated secretory phenotypes reveal cell-nonautonomous functions of oncogenic RAS and the p53 tumor suppressor. PLoS Biol 6: 2853-2868

Cortez DM, Feldman MD, Mummidi S, Valente AJ, Steffensen B, Vincenti M, Barnes JL, Chandrasekar B (2007) IL-17 stimulates MMP-1 expression in primary human cardiac fibroblasts via p38 MAPK- and ERK1/2-dependent C/EBP-beta, NF-kappaB, and AP-1 activation. Am J Physiol Heart Circ Physiol 293: H3356-H3365

Courtois-Cox S, Jones SL, Cichowski K (2008) Many roads lead to oncogene-induced senescence. Oncogene 27: 2801-2809

Cuenda A, Rouse J, Doza YN, Meier R, Cohen P, Gallagher TF, Young PR, Lee JC (1995) SB 203580 is a specific inhibitor of a MAP kinase homologue which is stimulated by cellular stresses and interleukin-1. FEBS Lett 364: 229-233

Cuenda A, Rousseau S (2007) p38 MAP-kinases pathway regulation, function and role in human diseases. Biochim Biophys Acta 1773: $1358-1375$

Davalos AR, Coppe JP, Campisi J, Desprez PY (2010) Senescent cells as a source of inflammatory factors for tumor progression. Cancer Metastasis Rev 29: 273-283

Davis T, Baird D, Haughton M, Jones CJ, Kipling D (2005) Prevention of accelerated cell aging in Werner syndrome using a p38 mitogen-activated protein kinase inhibitor. J Gerontol A Biol Sci Med Sci 60: 1386-1393

Davis T, Kipling D (2009) Assessing the role of stress signalling via p38 MAP kinase in the premature senescence of Ataxia Telangiectasia and Werner syndrome fibroblasts. Biogerontology 10: $253-266$

Defrance M, Touzet H (2006) Predicting transcription factor binding sites using local over-representation and comparative genomics. BMC Bioinformatics 7: 396

Deng Q, Liao R, Wu B-L, Sun P (2004) High intensity ras signalling induces premature senescence by activating p38 pathway in primary human fibroblasts. J Biol Chem 279: 1050-1059

Di Micco R, Fumagalli M, Cicalese A, Piccinin S, Gasparini P, Luise C, Schurra C, Garre M, Nuciforo PG, Bensimon A, Maestro R, Pelicci PG, d'Adda di Fagagna F (2006) Oncogeneinduced senescence is a DNA damage response triggered by DNA hyper-replication. Nature 444: 638-642

Dimri GP, Lee X, Basile G, Acosta M, Scott G, Roskelley C, Medrano EE, Linskens M, Rubelj I, Pereira-Smith O, Peacocke M, Campisi J (1995) A biomarker that identifies senescent human cells in culture and in aging skin in vivo. Proc Natl Acad Sci USA 92: 9363-9367

Eisen MB, Spellman PT, Brown PO, Botstein D (1998) Cluster analysis and display of genome-wide expression patterns. Proc Natl Acad Sci USA 95: 14863-14868

Enslen H, Raingeaud J, Davis RJ (1998) Selective activation of p38 mitogen-activated protein (MAP) kinase isoforms by the MAP kinase kinases MKK3 and MKK6. J Biol Chem 273: 1741-1748
Freund A, Orjalo AV, Desprez PY, Campisi J (2010) Inflammatory networks during cellular senescence: causes and consequences. Trends Mol Med 16: 238-246

Gil J, Peters G (2006) Regulation of the INK4b-ARF-INK4a tumour suppressor locus: all for one or one for all. Nat Rev Mol Cell Biol 7: $667-677$

Iwasa H, Han J, Ishikawa F (2003) Mitogen-activated protein kinase p38 defines the common senescence-signalling pathway. Genes Cells 8: 131-144

Janssens S, Tschopp J (2006) Signals from within: the DNA-damageinduced NF-kappaB response. Cell Death Differ 13: 773-784

Jeyapalan JC, Ferreira M, Sedivy JM, Herbig U (2007) Accumulation of senescent cells in mitotic tissue of aging primates. Mech Ageing Dev 128: $36-44$

Jun JI, Lau LF (2010) The matricellular protein CCN1 induces fibroblast senescence and restricts fibrosis in cutaneous wound healing. Nat Cell Biol 12: 676-685

Karin M (2006) Nuclear factor-[kappa]B in cancer development and progression. Nature 441: 431-436

Kortlever RM, Higgins PJ, Bernards R (2006) Plasminogen activator inhibitor-1 is a critical downstream target of p53 in the induction of replicative senescence. Nat Cell Biol 8: 877-884

Krizhanovsky V, Yon M, Dickins RA, Hearn S, Simon J, Miething C, Yee H, Zender L, Lowe SW (2008) Senescence of activated stellate cells limits liver fibrosis. Cell 134: 657-667

Krtolica A, Parrinello S, Lockett S, Desprez P-Y, Campisi J (2001) Senescent fibroblasts promote epithelial cell growth and tumorigenesis: a link between cancer and aging. Proc Natl Acad Sci USA 98: $12072-12077$

Kuilman T, Michaloglou C, Vredeveld LCW, Douma S, van Doorn R, Desmet CJ, Aarden LA, Mooi WJ, Peeper DS (2008) Oncogeneinduced senescence relayed by an interleukin-dependent inflammatory network. Cell 133: 1019-1031

Kwong J, Hong L, Liao R, Deng Q, Han J, Sun P (2009) p38alpha and p38gamma mediate oncogenic Ras-induced senescence through differential mechanisms. J Biol Chem 284: 11237-11246

Lali FV, Hunt A, Turner S, Foxwell B (2000) The pyridinyl imidazole inhibitor SB203580 blocks phosphoinositide-dependent protein kinase activity, protein kinase B phosphorylation, and retinoblastoma hyperphosphorylation in interleukin-2-stimulated $\mathrm{T}$ cells independently of p38 mitogen-activated protein kinase. J Biol Chem 275: 7395-7402

Liu D, Hornsby PJ (2007) Senescent human fibroblasts increase the early growth of xenograft tumors via matrix metalloproteinase secretion. Cancer Res 67: 3117-3126

Naldini L, Blomer U, Gage FH, Trono D, Verma IM (1996) Efficient transfer, integration, and sustained long-term expression of the transgene in adult rat brains injected with a lentiviral vector. Proc Natl Acad Sci USA 93: 11382-11388

Ohtani N, Yamakoshi K, Takahashi A, Hara E (2004) The p16INK4aRB pathway: molecular link between cellular senescence and tumor suppression. J Med Invest 51: 146-153

Ono K, Han J (2000) The p38 signal transduction pathway: activation and function. Cell Signal 12: 1-13

Orjalo AV, Bhaumik D, Gengler BK, Scott GK, Campisi J (2009) Cell surface-bound IL-1 $\{$ alpha\} is an upstream regulator of the senescence-associated IL-6/IL-8 cytokine network. Proc Natl Acad Sci USA 106: 17031-17036

Ossovskaya VS, Mazo IA, Chernov MV, Chernova OB, Strezoska Z, Kondratov R, Stark GR, Chumakov PM, Gudkov AV (1996) Use of genetic suppressor elements to dissect distinct biological effects of separate p53 domains. Proc Natl Acad Sci USA 93: 10309-10314

Paradis V, Youssef N, Dargere D, Ba N, Bonvoust F, Bedossa P (2001) Replicative senescence in normal liver, chronic hepatitis $\mathrm{C}$, and hepatocellular carcinomas. Hum Pathol 32: 327-332

Parrinello S, Coppe J-P, Krtolica A, Campisi J (2005) Stromalepithelial interactions in aging and cancer: senescent fibroblasts alter epithelial cell differentiation. J Cell Sci 118: 485-496

Passos JF, Nelson G, Wang C, Richter T, Simillion C, Proctor CJ, Miwa S, Olijslagers S, Hallinan J, Wipat A, Saretzki G, Rudolph KL, Kirkwood TB, von Zglinicki T (2010) Feedback between p21 and reactive oxygen production is necessary for cell senescence. Mol Syst Biol 6: 347

Perkins ND (2007) Integrating cell-signalling pathways with NF-kappaB and IKK function. Nat Rev Mol Cell Biol 8: 49-62

Prieur A, Peeper DS (2008) Cellular senescence in vivo: a barrier to tumorigenesis. Curr Opin Cell Biol 20: 150-155 
Radtke S, Wuller S, Yang XP, Lippok BE, Mutze B, Mais C, de Leur HS, Bode JG, Gaestel M, Heinrich PC, Behrmann I, Schaper F, Hermanns HM (2010) Cross-regulation of cytokine signalling: pro-inflammatory cytokines restrict IL-6 signalling through receptor internalisation and degradation. J Cell Sci 123: 947-959

Rodier F, Campisi J, Bhaumik D (2007) Two faces of p53: aging and tumor suppression. Nucleic Acids Res 35: 7475-7484

Rodier F, Coppe JP, Patil CK, Hoeijmakers WA, Munoz DP, Raza SR, Freund A, Campeau E, Davalos AR, Campisi J (2009) Persistent DNA damage signalling triggers senescenceassociated inflammatory cytokine secretion. Nat Cell Biol 11: 973-979

Rodier F, Munoz DP, Teachenor R, Chu V, Le O, Bhaumik D, Coppe J-P, Campeau E, Beausejour C, Kim S-H, Davalos AR, Campisi J (2011) DNA-SCARS: distinct nuclear structures that sustain damage-induced senescence growth arrest and inflammatory cytokine secretion. J Cell Sci 124: 68-81

Serrano M, Lin AW, McCurrach ME, Beach D, Lowe SW (1997) Oncogenic ras provokes premature cell senescence associated with accumulation of p53 and p16INK4a. Cell 88: 593-602

Sun P, Yoshizuka N, New L, Moser BA, Li Y, Liao R, Xie C, Chen J, Deng Q, Yamout M, Dong M-Q, Frangou CG, Yates JR, Wright PE, Han J (2007) PRAK is essential for ras-induced senescence and tumor suppression. Cell 128: 295-308

Wajapeyee N, Serra RW, Zhu X, Mahalingam M, Green MR (2008) Oncogenic BRAF induces senescence and apoptosis through pathways mediated by the secreted protein IGFBP7. Cell $\mathbf{1 3 2}$ 363-374
Wang SW, Pawlowski J, Wathen ST, Kinney SD, Lichenstein HS, Manthey CL (1999) Cytokine mRNA decay is accelerated by an inhibitor of p38-mitogen-activated protein kinase. Inflamm Res 48: $533-538$

Wang W, Chen JX, Liao R, Deng Q, Zhou JJ, Huang S, Sun P (2002) Sequential activation of the MEK-extracellular signal-regulated kinase and MKK3/6-p38 mitogen-activated protein kinase pathways mediates oncogenic ras-induced premature senescence. Mol Cell Biol 22: 3389-3403

Wilson KP, McCaffrey PG, Hsiao K, Pazhanisamy S, Galullo V, Bemis GW, Fitzgibbon MJ, Caron PR, Murcko MA, Su MS (1997) The structural basis for the specificity of pyridinylimidazole inhibitors of p38 MAP kinase. Chem Biol 4: 423-431

Xue W, Zender L, Miething C, Dickins RA, Hernando E, Krizhanovsky V, Cordon-Cardo C, Lowe SW (2007) Senescence and tumour clearance is triggered by p53 restoration in murine liver carcinomas. Nature 445: 656-660

Young PR, McLaughlin MM, Kumar S, Kassis S, Doyle ML, McNulty D, Gallagher TF, Fisher S, McDonnell PC, Carr SA, Huddleston MJ, Seibel G, Porter TG, Livi GP, Adams JL, Lee JC (1997) Pyridinyl imidazole inhibitors of p38 mitogen-activated protein kinase bind in the ATP site. J Biol Chem 272: 12116-12121

Zarubin T, Han J (2005) Activation and signalling of the p38 MAP kinase pathway. Cell Res 15: 11-18

Zhang J, Shen B, Lin A (2007) Novel strategies for inhibition of the p38 MAPK pathway. Trends Pharmacol Sci 28: 286-295

Zhou S, Greenberger JS, Epperly MW, Goff JP, Adler C, Leboff MS, Glowacki J (2008) Age-related intrinsic changes in human bonemarrow-derived mesenchymal stem cells and their differentiation to osteoblasts. Aging Cell 7: 335-343 